\title{
Allgemeine Wirkfaktoren in der schriftbasierten Onlinetherapie und -beratung
}

\author{
Gerhard Hintenberger
}

Angenommen: 22. November 2021 / Online publiziert: 9. Dezember 2021

(C) Der/die Autor(en) 2021

\begin{abstract}
Zusammenfassung Die ständig wachsenden technischen Möglichkeiten führen im Kontext von Onlinetherapie und Onlineberatung zu einer zunehmenden Ausdifferenzierung in unterschiedliche Kommunikationsmedien und verschiedene Settingumgebungen. Verschiedene Klient_innengruppen können so individuell angesprochen und auf deren Problemlagen präziser eingegangen werden. Andererseits wächst dadurch die Gefahr, digitalisierte Interventionen beliebig und eklektisch einzusetzen. Die Settingwahl ist deshalb als bewusste Interventionsstrategie anzusehen und benötigt ein Wissen über allgemeine Wirkfaktoren der unterschiedlichen Kommunikationskanäle, um eine Entscheidungsgrundlage für ein bestimmtes Setting oder einen Settingwechsel innerhalb eines Beratungs- und Therapiegeschehens treffen zu können. In diesem narrativen Review werden allgemeine Wirkfaktoren für die schriftbasierte Onlineberatung und Onlinetherapie identifiziert und als „Wirkfaktor Online-Beziehung“, „Wirkfaktor Sinneskanalreduktion“, „Wirkfaktor Anonymität“, „Wirkfaktor schriftbasierte Nachhaltigkeit“ sowie als „Wirkfaktor selbstreflexives und zeitautonomes Schreiben" kategorisiert.
\end{abstract}

Schlüsselwörter Onlineberatung · Onlinetherapie · Allgemeine Wirkfaktoren · Schriftbasierte Interventionen

Common factors in writing-based online therapy and online counseling

Summary The constantly growing technical possibilities available for both online therapy and online counselling have led to an increase of options within com-

\section{G. Hintenberger $(\bowtie)$}

Ringstraße 48, 3500 Krems, Österreich

info@praxis-hintenberger.at munication medias and setting environments. Groups of clients may be addressed individually and their problems dealt with more precisely. However, this increases the risks of using digitalised interventions both arbitrarily and eclectically. The choice of setting should therefore be seen as a conscious intervention strategy and will require knowledge surrounding the different communication channels in order to be able to make appropriate decisions that may affect a setting or a change of setting within the counselling and therapy process. In this narrative review, common factors of both writing-based online counselling and online therapy are identified and categorised as "online relationship", "sensory channel reduction", "anonymity", "writing-based sustainability" and as "self-reflexive and time-autonomous writing".

Keywords Online counselling · Online therapy · Common factors · Writing-based interventions

\section{Einleitung}

Universitäten verfügten schon zu einem sehr frühen Zeitpunkt über computerbasierte Ressourcen und so konnte bereits 1986, also noch Jahre vor der Implementierung des World Wide Web, an der Cornell University in den USA die Onlineberatungsstelle „Dear Uncle Ezra“ (benannt nach dem Universitätsgründer Ezra Cornell) ihren Betrieb aufnehmen. Der ehemalige Direktor des universitären Beratungszentrums Jerry Feist hatte die Idee, dass es den Studierenden leichter fallen könnte, anonymisiert und schriftlich ihre Probleme zu kommunizieren (Grohol 2010). Da sich studentische Problemlagen ähneln, wurden sowohl Anfragen als auch Antworten für alle sichtbar veröffentlicht, in der Hoffnung, dass darüber hinaus auch Mitleser_innen von diesen Dialogen profitieren konnten. 
Im deutschsprachigen Raum gehörte die Telefonseelsorge, die 1995 mit ihren Angeboten online ging, zu den Early Adopters (Knatz 2013). Onlineberatung und -therapie gab es also schon lange vor der Coronapandemie, auch wenn situationsbedingt seither eine breite Palette unterschiedlicher Onlineanwendungen $\mathrm{zu}$ beobachten ist, nicht selten unter Umgehung von Qualitäts- und Datenschutzkriterien. Diese Formate blicken auf eine über 30jährige Geschichte zurück und sehen sich seit einigen Jahren mit einer zunehmenden Ausdifferenzierung in unterschiedliche Kommunikationsmedien und -modi sowie verschiedene Settingumgebungen konfrontiert.

Dies führt zu erweiterten Möglichkeiten in Bezug auf unterschiedliche Klient_innengruppen und deren Problemlagen. Auf der anderen Seite wächst aber auch die Gefahr einer unreflektierten, eklektisch ausgerichteten Settingflexibilität sowie einer damit einhergehenden Beliebigkeit des Einsatzes digitalisierter Interventionen. Ein Wissen über Wirkfaktoren unterschiedlicher Kommunikationskanäle kann eine wesentliche Entscheidungsgrundlage für die Kanalwahl innerhalb eines Beratungs- und Therapiegeschehens bilden. Der vorliegende Artikel beschäftigt sich als narrativer Review mit der Frage, welche allgemeinen Wirkfaktoren für eine schriftbasierte Onlineberatung und -therapie in Forschung und Literatur genannt werden. Ziel ist es, am Beispiel der schriftbasierten Onlineberatung durch eine Kategorisierung dieser Wirkfaktoren einen präziseren Einsatz von unterschiedlichen Settingsvarianten und den dazu gehörenden Onlineinterventionen zu ermöglichen.

Grundlage für den vorliegenden narrativen Review bieten deutschsprachige Lehrbücher und Kompendien zu Onlineberatung und Onlinetherapie (siehe Tab. 1). Die Lehrbücher und Kompendien wurden auf dort implizit und explizit genannte Wirkfaktoren hin untersucht und diese in der Folge kategorisiert. Lehrbuchartige Überblickswerke finden sich fast ausschließlich im deutschsprachigen, nicht jedoch im englischsprachigen Raum. Eine selektive Literatursuche in den Datenbanken Psyndex, Pubmed und biber/EBESCO mit den Suchbegriffen „Onlineberatung“ UND (boolesche Operator) „Wirkfaktoren“ bzw. „Onlinetherapie“ UND „allgemeine Wirkfaktoren“ sowie „online counseling“ AND „common factors“ bzw. „on- line therapy“ AND „common factors“ brachte keine Ergebnisse. Eine Kategorisierung allgemeiner Wirkfaktoren entlang empirischer Befunde kann deshalb zum jetzigen Zeitpunkt nicht erfolgen. $\mathrm{Zu}$ erwähnen ist noch eine aktuelle Dissertation (Jonas 2020) zu „Wirkfaktoren internetbasierter Beratungsinterventionen“.

\section{Definitionen}

Sowohl im deutschsprachigen als auch im englischsprachigen Umfeld gibt es inzwischen eine inflationäre Bezeichnungsvielfalt, die von distance therapy, e-therapy, internet-based treatment, computer intervention über e-counseling und computer counseling bis hin zu Onlineberatung, Onlinetherapie oder internetbasierte Interventionen reicht. In einer universitäten- und länderübergreifenden Erklärung machten 22 klinische Expert_innen und Forscher_innen auf die Schwierigkeiten einer inkonsistenten Terminologie bei internetgestützten Interventionen aufmerksam (Smoktunowicz et al. 2020). Die Auswirkungen dieser Vielgestaltigkeit betreffen unterschiedliche Ebenen wie Literaturrecherche oder die Frage inwieweit die eingesetzte Technologie als Teil der Intervention oder nur in Bezug auf ihre Fähigkeit zur Bereitstellung von Kommunikationsphänomenen betrachtet werden muss. Die Autor_innen schlagen deshalb die Bildung eines Expert_innenteams vor, um eine konsensuale Erklärung zur Implementierung eines einheitlichen Glossars vorzubereiten (Smoktunowicz et al. 2020).

Der vorliegende Artikel konzentriert sich auf den schriftbasierten Bereich internetgestützter Interventionen, die sich sowohl methodisch als auch zielgruppenspezifisch vielgestaltig darstellen und sich in Form von Blended Counseling/Therapy und Standalone-Ansätzen settingvariabel präsentieren (Reindl 2018). Die Unterteilung von synchronen und asynchronen Kommunikationsmedien, wie sie auch die Internetrichtlinie des österreichischen Ministeriums für Soziales, Gesundheit, Pflege und Konsumentenschutz präferiert (2020), wird den technischen Möglichkeiten diverser Medien nicht mehr gerecht. Fluide Kommunikationskanäle, wie zum Beispiel Messengerdienste, können anlassbezogen sowohl synchron als auch asynchron sowie schrift- video- oder audioba-

Tab. 1 Deutschsprachige Lehrbücher und Kompendien zu Onlineberatung und -therapie

Knatz, Birgit \& Dodier, Bernard (2021). Mailen, chatten, Engelhardt, Emily M. (2021). zoomen: Digitale Beratungsformen in der Praxis Justen-Horsten, Agnes \& Paschen, Helmut (2016). Online-Interventionen in Therapie und Beratung. Ein Praxisleitfaden

Eichenberg und Kühne (2014). Einführung Onlineberatung \& -therapie. Grundlagen, Interventionen und Effekte digitaler Medien

Ploil, Eleonore 0ja (2009). Psychosoziale Onlineberatung Lehrbuch Onlineberatung Berger, Thomas (2015). Internetbasierte Interventionen bei psychischen Störungen

Wenzel, Joachim (2013). Wandel der Beratung durch Neue Medien

Kühne und Hintenberger (2009). Handbuch Online-Beratung
Knaevelsrud et al. (2016). Online-Therapie und -Beratung. Ein Praxisleitfaden zur onlinebasierten Behandlung psychischer Störungen

Krieger, Alfred, Winter, Heike, Müller, Ulrich A., Ochs, Matthias \& Broicher, Wiebke (2015). Geht die Psychotherapie ins Netz? Möglichkeiten und Probleme von Therapie und Beratung im Internet

Geißler, Harald \& Metz, Maren (2012). E-Coaching und Online-Beratung. Formate, Konzepte, Diskussionen

Bauer, Stephanie \& Kordy, Hans (2008). E-Mental-Health. Neue Medien in der psychosozialen Versorgung

Knatz und Dodier (2003). Hilfe aus dem Netz. Theorie und Praxis der Beratung per E-Mail 
siert eingesetzt werden. Eine strikte Trennung dieser Dienste nach zeitlichen oder sinneskanalorientierten Dimensionen ist schon seit einigen Jahren nicht mehr möglich. Auf eine vertiefende Abgrenzungsdiskussion von Onlineberatung und Onlinetherapie, die sowohl inhaltlich-methodische als auch juristische Bereiche umfassen müsste, wird hier verzichtet. Die Trennlinie wird pragmatisch beim Vorliegen einer Diagnose zur Behandlung von krankheitswertigen Symptomen als Voraussetzung für eine Onlinetherapie gezogen.

\section{Schriftbasierte Onlinetherapie und-beratung}

Auffallend ist zunächst einmal der gehäufte Einsatz von Manualen aus dem Umfeld der Präsenz-Verhaltenstherapie mit kognitiv-behavioralem Schwerpunkt innerhalb eines schrift- und internetbasierten Settings. Diese weisen oftmals eine stärkere Strukturierung auf, als dies in der Präsenztherapie der Fall ist, können deshalb manualgetreuer umgesetzt und in der Folge präziser beforscht werden (Knaevelsrud et al. 2016). Am bekanntesten davon ist das hochstrukturierte Behandlungsprogramm „Interapy“, das ursprünglich zur Behandlung von posttraumatischen Belastungsstörungen entwickelt, inzwischen aber für zusätzliche Störungsbilder erweitert wurde (Berger 2015; Wagner und Lange 2008). „Interapy“ und ähnlich aufgebaute Behandlungsangebote sind inzwischen gut evaluiert, weisen eine solide empirische Evidenz auf (Eichenberg 2021) und erzielen ähnliche gute Behandlungseffekte wie manualisierte kognitiv-behaviorale Präsenztherapien (Klasen et al. 2013; Knaevelsrud et al. 2016).

Um individuelle Variablen stärker in den Blick nehmen und Interventionen präziser einsetzen zu können, kommen vermehrt auch personalisierte InternetPsychotherapie-Angebote (Maercker et al. 2015) sowie transdiagnostische Tools zur Anwendung (Knaevelsrud et al. 2016). Webbasierte psychodynamische Interventionen wurden erst in den letzten Jahren entwickelt und zeigen moderate bis gute Effekte (Zwerenz et al. 2017). Im Bereich der Onlineberatung werden vor allem prozessorientierte Modelle aus dem klientzentrierten (Knatz und Dodier 2003), systemischen (Engelhardt 2021) und integrativen (Hintenberger 2019) Umfeld adaptiert, wobei es bislang nur vereinzelte Evaluierungen von internetbasierten Beratungsangeboten gibt, die jedoch durchaus positive Ergebnisse zeigen (Eichenberg und Aden 2015; Eichenberg 2007).

Jonas (2020) kritisiert, dass Forschung zu internetbasierten Interventionen noch immer zu selten die besonderen Nutzungsbedingungen dieses Settings in den Blick nimmt und stattdessen davon ausgeht, dass ähnliche Wirkmechanismen zum Tragen kommen wie in einer Face-to-Face-Umgebung. Ob der Einsatz spezifischer Interventionen innerhalb eines Settings, das einerseits eine radikale Sinneskanalreduktion zur Folge hat und andererseits ausschließlich einen schriftli- chen Kommunikationskanal zur Verfügung stellt, ohne nennenswerte Auswirkungen bleibt, ist tatsächlich fraglich. Es lohnt sich also in diesem Zusammenhang einen Blick auf die allgemeinen Wirkfaktoren zu werfen und die bestimmenden Variablen „schriftlich“ und „internetbasiert“ auf ihre Einflussfaktoren hin zu untersuchen.

\section{Wirkfaktoren}

Die Hauptfrage dieser Arbeit beschäftigt sich mit allgemeinen Wirkfaktoren schrift- und internetbasierter Interventionen. Die Rede ist hier von Therapie- und Beratungsvariablen, die als inhärenter Bestandteil innerhalb therapeutischer Interaktionen auftreten und dabei abseits spezifischer, meist symptomindizierter Interventionen wirksam werden (Pfammatter et al. 2012). Es geht also nicht darum, spezielle Interventionstechniken, die online angewandt werden, auf ihre Wirksamkeit hin zu überprüfen, sondern Literatur und Forschung dahingehend $\mathrm{zu}$ befragen, ob das online- und schriftbasierte Setting allgemeine Wirkfaktoren zur Verfügung stellt. Mit Pfammatter et al. (2012) soll hier nochmals betont werden, dass die Diskussion um allgemeine und spezifische Wirkfaktoren oftmals unnötig polarisiert geführt wird, da sie nicht als isoliert, sondern nur innerhalb eines gemeinsamen Kontextes betrachtet werden können. So ist zum Beispiel „Schreiben als Konfrontation“ ein gut evaluierter spezifischer Wirkfaktor im Rahmen einer internetbasierten Therapie für Traumapatient_innen (Knaevelsrud et al. 2016), der aber im Rahmen der allgemeinen Wirkfaktoren „Sinneskanalreduktion“ sowie „zeitversetzte Kommunikation“ seine besondere Geltung erlangt. Huibers et al. (2021) betonen zudem, dass es bis heute noch keine zwingende Evidenz für allgemeine oder spezifische Faktoren gibt, die vorrangig eine Veränderung in der Psychotherapie bewirken.

\section{Allgemeiner Wirkfaktor "Online-Beziehung"}

Das Beziehungsgeschehen in der schriftbasierten Kommunikation läuft durch den Wegfall von nonverbalen und paraverbalen Phänomenen unter besonderen Bedingungen ab. Die Skepsis, dass dadurch keine hinreichend gute therapeutische Beziehung aufgebaut werden kann, wird von der aktuellen Studienlage nicht bestätigt. Knaevelsrud et al. (2016) fassen vorliegende systematische Reviews dahingehend zusammen, dass internetbasierte Kommunikation in einer Onlinetherapie von gegenseitiger Akzeptanz und Zufriedenheit geprägt ist und der Kontakt als angenehm und persönlich erlebt wird. Auch Berger (2017) konstatiert in einem narrativen Review, dass es unabhängig von Setting, Diagnosen und zeitlichem Umfang vergleichbare Allianzwerte aus Sicht der Klient_innen gibt.

Die Qualität therapeutischer Beziehungen wird von Seiten der Klient_innen in der Regel entlang 
ihrer Alltagserfahrungen beurteilt und in Kategorien wie Sympathie, verstehendes Zuhören, Vertrauen, Empathie, Respekt und der Kompetenz, Lösungsmöglichkeiten zu vermitteln, beschrieben (Hermer und Röhrle 2008). Da private Beziehungen heute in einem zunehmenden Ausmaß auch über internetbasierte Kommunikationskanäle realisiert werden, verwundert es nicht, dass Klient_innen diese Erfahrungswerte zur Einschätzung der Beziehungsqualität in einer Onlineberatung- oder therapie heranziehen (Gahleitner und Preschl 2016).

Inwieweit die Arbeitsallianz ähnlich wie bei Faceto-Face-Therapien mit dem Therapieerfolg korreliert, wird durch vorliegende Studien allerdings bislang nicht einheitlich beantwortet. Da vor allem in der asynchronen Onlinetherapie und -beratung die Eigenständigkeit der Klient_innen stärker gefordert ist, werden Symptomverbesserungen tendenziell eher sich selbst als den Therapeut_innen oder der Therapiebeziehung zugeschrieben (Jonas 2020; Andersson 2016). Dies kann durchaus auch als eigenständiger Wirkfaktor angesehen werden, da in diesem Fall Klient_innen Therapiefortschritte vor allem im Kontext ihrer eigenen Selbstwirksamkeit sehen.

Probst et al. (2019) kommen in ihrer Metaanalyse hingegen zu dem Schluss, dass der Arbeitsallianz sehr wohl eine ähnlich bedeutsame Rolle wie in der Faceto-Face-Therapie zukommt, wenn auch eine Identifikation mit vorgegebenen Aufgabenstellungen einen etwas größeren Einflussfaktor für den Therapieerfolg $\mathrm{zu}$ haben scheint als die emotionale Bindung an den_die Therapeut_in. Die Autor_innen erklären sich diesen Effekt, der trotz der Einschränkungen einer sinneskanalreduzierten, ausschließlich schriftbasierten Kommunikation zu Stande kommt, mit einem qualitativ-konzeptuell unterschiedlichen Beziehungskonzept als in einer Face-to-Face-Therapie: Das Fehlen nonverbaler und paraverbaler Phänomene wird durch spezielle Kommunikationselemente neu codiert und damit zumindest $\mathrm{zu}$ einem Teil ausgeglichen. Die nicht vorhandene physische Co-Präsenz ermöglicht eine idealisierend-projektive Gestaltung des Gegenübers nach eigenen Vorstellungen sowie ein höheres Maß an Offenheit durch die Möglichkeit, nur schriftlich miteinander zu kommunizieren (Probst et al. 2019). Der Einflussfaktor Beziehung zeigt sich auch darin, dass sich in der Wirksamkeit schriftbasierter, aber therapeut_innenunterstützter sowie ungeleiteter Selbsthilfeangebote signifikante Unterschiede zugunsten therapeut_innenunterstützter Prozesse zeigen (Berger 2015).

Ähnliches gilt auch für Onlineberatungsangebote, wo dem Faktor „Beratungsbeziehung“ schon alleine wegen des weniger strukturierten Vorgehens ein größerer Einfluss zugeschrieben wird als spezifischen Interventionen (Eichenberg und Aden 2015; Eichenberg 2007). Notwendig ist jedenfalls eine entsprechende Schulung, um die Fachkräfte mit den Besonderheiten der Onlinekommunikation vertraut zu machen (Gahleitner und Preschl 2016).

\section{Allgemeiner Wirkfaktor „Sinneskanalreduktion“}

Nicola Döring (2000) verweist schon zu einem sehr frühen Zeitpunkt in der Theoriebildung zur Kommunikation im Internet darauf, dass eine Sinneskanalreduktion keineswegs automatisch mit einer Verarmung von Empfindungen und Gefühlen einhergehen muss, sondern $\mathrm{zu}$ einer „Dialektik von körperlicher Abwesenheit und sinnlicher Präsenz, physischer Distanz und psychologischer Nähe" (Döring 2000, S. 367) führt. Sinneskanalreduktion auf der einen Seite führt automatisch zu einer Sinneskanalfokussierung auf einer anderen Ebene. Dies ist ein bekannter Effekt, der zum Beispiel auch bei Menschen mit einer visuellen Beeinträchtigung zu beobachten ist und eine Sensibilisierung im Hörbereich sowie in der haptischen Wahrnehmung nach sich ziehen kann. Das Ausschalten visueller und akustischer Reize öffnet zudem projektive Räume, die eine schnellere Selbstöffnung bewirken. Klient_innen müssen sich nicht tatsächlichen oder phantasierten Bewertungen durch Berater_innen in einem Face-to-Face-Umfeld aussetzen und können dadurch im wahrsten Sinne des Wortes ihr Gesicht wahren (Schultze 2007).

\section{Allgemeiner Wirkfaktor „Anonymität“}

Die Auswirkungen dieses Wirkfaktors sind vielfältig und stehen in einem engen Zusammenhang mit dem Wirkfaktor „Online-Beziehung“. In den meisten Lehrbüchern und Kompendien (Eichenberg und Kühne 2014; Engelhardt 2021; Knaevelsrud et al. 2016; Knatz und Dodier 2003; Kühne und Hintenberger 2009) wird die Tatsache betont, dass schriftbasierte Onlineberatung eine offenere Problemkommunikation erleichtert, da die Ratsuchenden den Grad der Anonymität selbst bestimmen können und in der Gestaltung des Beratungsprozesses über mehr Autonomie verfügen als in einer Face-to-Face-Beratung. Suler (2004) spricht in diesem Zusammenhang von einem „disinhibition effect", wobei Klient_innen durch die Reduktion von Schamgefühlen schneller und präziser relevante sowie angstbesetzte Themen kommunizieren können.

Eichenberg und Aden (2015) bestätigen diese Effekte in der Evaluierung eines Onlineberatungsangebots bei Partnerschaftskonflikten und psychosozialen Krisen. Klient_innen erleben dabei den Aufbau von Vertrautheit und Nähe schneller als gewohnt und fühlen sich durch die mediale Distanz besser geschützt. Die parallel dazu befragten Berater_innen nehmen die Vorteile dieses Settings wahr, zeigen sich aber durch die größeren Möglichkeiten in Bezug auf Autonomie und Beziehungsgestaltung auf Seiten der Klient_innen auch verunsichert. Betrachtet man die Anlassproblematiken, die zur Inanspruchnahme einer Onlinebera- 
tung führen, so fällt der relative hohe Anteil an schambesetzten Themen auf, wobei auch Klient_innen, die dezidiert Onlineberatungsangebote nutzen über eine klinisch relevante Symptomatik verfügen (Eichenberg und Aden 2015).

Klient_innen sind also durch die vielfältigen Möglichkeiten dieser Medien zu selbstbestimmter Kontaktsteuerung sowohl in der Lage, eigenständig Schutzräume herzustellen als auch schneller Selbstöffnungsund Problemkommunikationsprozesse zu realisieren.

\section{Allgemeiner Wirkfaktor „Schriftbasierte Nachhaltigkeit"}

Die schriftliche Darstellung in Form von Mail-, Messenger- und Chatverläufen ermöglicht eine Vertiefung des Problemverständnisses und der Informationsaneignung, da die Aussagen der Berater_innen nachhaltig verfügbar sind und so wiederholt gelesen werden können. Wichtige Informationen bleiben archiviert erhalten und „verhallen“ nicht, wie dies während und nach einer akkustikzentrierten Face-to-Face-Beratung passiert. Digitalisierte Speicherung schriftbasierter Dialoge sowie die Möglichkeit einer mehrstufigen Textaneignung erhöhen die Transferwahrscheinlichkeit der Beratungsinhalte in den Lebensalltag der Klient_innen (Schultze 2007).

Wagner et al. (2014) konnten in einer randomisierten Studie bei Patient_innen mit einer mittelgradigen Depression die Gleichwertigkeit einer psychotherapeutischen Behandlung mit kognitiv-verhaltenstherapeutischen Interventionen in einem Faceto-Face bzw. Online-Setting belegen. In beiden Patient_innengruppen sanken die Depressionswerte signifikant. Eine katamnestische Überprüfung drei Monate nach Abschluss der Therapie zeigte, dass in der Onlinegruppe die Depressionswerte weiter zurück gingen, während es in der Face-to-Face-Gruppe zu minimalen Rückfällen kam. Die Autor_innen interpretieren dies mit einem höheren Fokus auf Selbstverantwortung, die durch eine geringere persönliche Begleitung und einem höheren schriftbasierten Hausaufgabenanteil entstanden ist (Wagner et al. 2014). Da die Patient_innen der Onlinegruppe aber auch angaben, die Korrespondenz mit den Therapeut_innen wiederholt gelesen zu haben, liegt es nahe, die niedrigeren Depressionswerte auch in einem Zusammenhang mit dem Wirkfaktor der „schriftbasierten Nachhaltigkeit“ zu sehen.

\section{Allgemeiner Wirkfaktor „selbstreflexives und zeitautonomes Schreiben"}

Dieser Wirkfaktor kommt sowohl in der manualisierten Onlinetherapie als auch in der prozessorientierten Onlineberatung zum Tragen, setzt aber eine asynchrone Settingumgebung voraus. Beim Verfassen einer Mail bzw. einer Messengernachricht oder auch bei der Umsetzung einer Schreibaufgabe erfolgt die
Auseinandersetzung zunächst einmal als Interaktion mit sich selbst (Knatz und Dodier 2003). Die eigenen Gedanken treffen nicht sofort auf die Reaktion eines Gegenübers und es ist nicht notwendig, zeitnahe auf Aussagen des_der Kommunikationspartner_in reagieren zu müssen (Suler 2004). Es entsteht eine „zone for reflection“ (Eichenberg und Kühne 2014). Ein asynchrones Setting unterstützt also die Selbstwirksamkeit und Eigenständigkeit der Ratsuchenden und wirkt damit einer strukturellen Asymmetrie und Klientifizierung entgegen (Schulze 2007).

Die so angeregten selbstreflexiven Prozesse führen automatisch in eine exzentrische Position, die den Klient_innen eine Problemdistanzierung, meist einen ersten Überblick und im günstigsten Falle sogar einen Expert_innenblick ermöglichen. Dies erweist sich vor allem in der Krisenintervention als hilfreich (HoferMoser et al. 2020).

\section{Diskussion}

Betrachtet man aktuelle Tendenzen im psychotherapeutischen Feld, dann zeigt sich in den letzten Jahren eine Hinwendung zu individualisierten (Sack 2019), personalisierten (Rubel und Lutz 2016), präzisionsorientierten (Meinlschmidt und Tegethoff 2017) Ansätzen bzw. Interventionen. Im Umfeld der Verhaltenstherapie lösen zunehmend modulare Sequenzen, die forschungsbasiert je nach Klient_innenpersönlichkeit, Therapiesituation und Störungsbild transdiagnostisch zum Einsatz kommen manualisierte Vorgehensweisen ab (Bohus et al. 2012).

Dass gerade internetbasierte Verfahren vielfältige Möglichkeiten der Individualisierung und Passfähigkeit für unterschiedliche Patient_innen bereitstellen, beschreiben Maercker et al. (2015) in einer Überblicksarbeit für die posttraumatische Belastungsstörung. Wer von belastenden Ereignissen betroffen ist, nutzt oftmals intuitiv die schriftliche Darlegung des Erlebten als erste Möglichkeit der Entlastung (Pennebaker 1997). Internetbasierte Angebote können hier bereits in einem Frühstadium der Behandlung wirksam werden und durch die Möglichkeit traumabezogene Inhalte schriftlich zu erzählen, einer Fragmentierung traumabezogener Gedächtnisinhalte entgegenwirken.

Durch die ständige Ausdifferenzierung der Settingvarianten in neue internetbasierte und virtuelle Angebote gewinnt die Settingwahl also zunehmend den Stellenwert einer Intervention. Wie am Beispiel der schriftbasierten Onlineberatung- und therapie dargelegt wurde, lassen sich für dieses Setting allgemeine Wirkfaktoren definieren, die persönlichkeitsbezogen und transdiagnostisch eingesetzt werden können.

- Trotz einer Reduktion der Sinneskanäle im schriftbasierten Setting fällt dem Wirkfaktor „Onlinebeziehung“ eine zentrale Rolle zu. Dies zeigt sich unter anderem an signifikanten Unterschieden in 
der Wirksamkeit zugunsten therapeutengeleiteter Selbsthilfeprogramme. Die proaktive Gestaltung der Beratungsbeziehung ist deshalb auch im schriftbasierten Onlinekontext als vorrangig zu betrachten.

- Der Wirkfaktor „Sinneskanalreduktion“ ermöglicht Patient_innen mit traumatisierenden Beziehungserfahrungen eine differenzierte Ausgestaltung von Nähe und Distanz im Sinne einer selbstwirksamen Wahrung der eigenen Grenzen.

- Schambesetzte Themen können ganz offensichtlich mit Hilfe des Wirkfaktors „Anonymität“ besser kommuniziert werden. Klient_innen verfügen in diesem Setting über mehr Möglichkeiten, sich einen eigenen Schutzraum für eine offene Problemkommunikation zu gestalten. Vor allem der Chat bietet hier die Möglichkeit, ein spontan- wechselseitiges Kommunikationsgeschehen mit Anonymisierungsstrategien zu verknüpfen.

- Mail- und Messengertexte können gezielt als Übergangsobjekte sowie in der Krisenintervention zur Stabilisierung eingesetzt eingesetzt werden. Die Texte stehen „nachhaltig“ zur Verfügung und können wiederholt gelesen werden. Um den Wirkfaktor „schriftbasierte Nachhaltigkeit“ zu verstärken, werden die Antworten in einem interkationsorientierten Schreibstil verfasst und dadurch beim Lesen „hörbar“ (Hintenberger und Engels, in Druck; Kühne und Hintenberger 2020). Diese besondere Form der schriftbasierten Nachhaltigkeit ermöglicht trotz asynchroner Kommunikation, die imaginierte Präsenz von Therapeut_innen und Berater_innen.

- Der Wirkfaktor „selbstreflexives und zeitautonomes Schreiben“ kommt vor allem Klient_innen zugute, die über genügend Ressourcen verfügen, um durch fokussierte Anregungen und Interventionen eigenständige Reflexionsprozesse in Gang zu setzen. Zudem unterstützt dieser Wirkfaktor Patient_innen in akuten Krisen auch zu Zeiten, in denen Berater_innen und Therapeut_innen nicht erreichbar sind.

Mit Ausnahme der Beforschung des Wirkfaktors „Onlinebeziehung“ lag der Fokus bislang in erster Linie auf Outcome-Forschung. Notwendig sind deshalb Forschungsansätze, die allgemeine Wirkfaktoren internetbasierter Beratung und Therapie präzisieren und untersuchen, wie sich eine Kombination verschiedener Settingvarianten innerhalb einer Behandlungssequenz auswirken. Um einem unreflektierten Einsatz unterschiedlicher Settings entgegen zu wirken, braucht es zudem einen Einbezug in bestehende Integrationsmodelle mit definierten Integratoren (Hintenberger et al. 2018; Sieper 2010). Ziel ist also eine systematische Nutzung unterschiedlicher Kommunikationskanäle, um Beratungs- und Therapieprozesse für Klient_innen und Patient_innen empirisch fundiert zu gestalten und zu nutzen. Neben einer Loslösung von impliziten Ideologismen (siehe „Mythos Unmittelbarkeit“ (Wenzel 2015)) ist auch eine Anpas- sung der rechtlichen Grundlagen, die sowohl von den technischen Möglichkeiten als auch von der gelebten Praxis längst überholt wurden, notwendig.

Interessenkonflikt G. Hintenberger gibt an, dass kein Interessenkonflikt besteht.

Open Access Dieser Artikel wird unter der Creative Commons Namensnennung 4.0 International Lizenz veröffentlicht, welche die Nutzung, Vervielfältigung, Bearbeitung, Verbreitung und Wiedergabe in jeglichem Medium und Format erlaubt, sofern Sie den/die ursprünglichen Autor(en) und die Quelle ordnungsgemäß nennen, einen Link zur Creative Commons Lizenz beifügen und angeben, ob Änderungen vorgenommen wurden.

Die in diesem Artikel enthaltenen Bilder und sonstiges Drittmaterial unterliegen ebenfalls der genannten Creative Commons Lizenz, sofern sich aus der Abbildungslegende nichts anderes ergibt. Sofern das betreffende Material nicht unter der genannten Creative Commons Lizenz steht und die betreffende Handlung nicht nach gesetzlichen Vorschriften erlaubt ist, ist für die oben aufgeführten Weiterverwendungen des Materials die Einwilligung des jeweiligen Rechteinhabers einzuholen.

Weitere Details zur Lizenz entnehmen Sie bitte der Lizenzinformation auf http://creativecommons.org/licenses/by/4. $0 /$ deed.de.

\section{Literatur}

Andersson, G. (2016). Internet-delivered psychological treatments. Annual Review of Clinical Psychology, 12(1), 157-179. https://doi.org/10.1146/annurev-clinpsy021815-093006.

Bauer, S. \& Kordy, H. (Hrsg.). (2008). E-Mental-Health. Neue Medien in der psychosozialen Versorgung. Heidelsberg: Springer Medizin Verlag

Berger, T. (2015). Internetbasierte Interventionen bei psychischen Störungen. Göttingen: Hogrefe.

Berger, T. (2017). The therapeutic alliance in internet interventions: a narrative review and suggestions for future research. Psychotherapy Research, 27(5), 511-524.https:// doi.org/10.1080/10503307.2015.1119908.

Bohus, M., Herpertz, S. C., \&Falkai, P. (2012). Modulare Psychotherapie - Rationale und Grundprinzipien. Die Psychiatrie, 9(2), 89-97.

Bundesministerium für Soziales, Gesundheit, Pflege und Konsumentenschutz (BMSGPK) (2020). Internetrichtlinie. Kriterien zur Ausgestaltung der psychotherapeutischen Beratung via Internet. https://www.sozialministerium. at/dam/jcr:48e371db-49fe-403f-b27f-c35dc949b21b/ Internetrichtlinie_(BMSGPK),_Stand_03.03.2020.pdf. Zugegriffen: 19. Juni 2021.

Döring, N. (2000). Kommunikation im Internet: Neun theoretische Ansätze. In B. Batinic (Hrsg.), Internet für Psychologen (2.Aufl. S. 345-377). Göttingen:Hogrefe.

Eichenberg, C. (2007). Online-Sexualberatung: Wirksamkeit und Wirkweise. Zeitschrift für Sexualforschung, 20(3), 247-262.https://doi.org/10.1055/s-2007-981238.

Eichenberg, C. (2021). Onlinepsychotherapie in Zeiten der Coronapandemie. Psychotherapeut, 66(3), 195-202.https:// doi.org/10.1007/s00278-020-00484-0.

Eichenberg, C., \& Aden, J. (2015). Onlineberatung bei Partnerschaftskonflikten und psychosozialen Krisen. Multimethodale Evaluation eines E-Mail-Beratungsangebots. Psy- 
chotherapeut, 60,53-63.https://doi.org/10.1007/s00278014-1092-y.

Eichenberg, C., \& Kühne, S. (2014). Einführung Onlineberatung \& -therapie. Grundlagen, Interventionen und Effekte digitaler Medien. Stuttgart:UTB.

Engelhardt, E. M. (2021). Lehrbuch Onlineberatung (2. Aufl.). Göttingen: Vandenhoeck\& Ruprecht.

Gahleitner, S. B., \& Preschl, B. (2016). Professionelle Beziehungsgestaltung über das Internet: Geht denn das überhaupt? Überlegungen zu einem methodenübergreifenden Wirkfaktor. Resonanzen. E-Journal für biopsychosoziale Dialoge in Psychotherapie, Supervision und Beratung, 4(2), 108-129. https://www.resonanzen-journal. org/index.php/resonanzen/article/view/392. Zugegriffen: 19. Juni 2021.

Geißler, H. \& Metz, M. (Hrsg.) (2012). E-Coaching und OnlineBeratung. Formate, Konzepte, Diskussionen. Wiesbaden: SpringerVS

Grohol, J. M. (2010). From Netscape to Twitter: Fifteen years of mental healthonline. In A. Brunet, A.R. Ashbaugh \& C. F. Herbert (Hrsg.), Internet use in the aftermath of trauma (S.3-22).Amsterdam:IOSPress.

Hermer, M., \& Röhrle, B. (2008). Therapeutische Beziehungen: Geschichte, Entwicklungen und Befunde. In M. Hermer \& B. Röhrle (Hrsg.), Allgemeiner Teil (Bd. 1, S. 15-105). Tübingen: dgvt.

Hintenberger, G. (2019). Prozessmodelle für die schriftbasierte Onlineberatung. e-beratungsjournal.net. Fachzeitschrift für Onlineberatung und computervermittelte Kommunikation 15(1), 26-37. http://www.e-beratungsjournal. net/wp-content/uploads/2019/03/hintenberger_2019_ 01.pdf.Zugegriffen: 19. Juni2021.

Hintenberger, G., \& Engels, S. (in Druck). Dialogorientierter Schreibstil und inszenierte Mündlichkeit, interaktionsorientiertes Schreiben sowie Textformen-basierte Interaktion in der Onlineberatung. e-beratungsjournal.net. Fachzeitschrift für Onlineberatung und computervermittelte Kommunikation

Hintenberger, G., Gahleitner, S. B., \& Pasch, S. (2018). Methodenintegration in Beratung und Psychotherapie. Zeitschrift für Psychodrama und Soziometrie, 17(2), 247-259. https://rdcu.be/38rr.Zugegriffen: 19. Juni2021.

Hofer-Moser, O., Hintenberger, G., Schwarzmann, M., \& Brunner, F. (2020). Krisenintervention kompakt. Theoretische Modelle, praxisbezogene Konzepte und konkrete Interventionsstrategien. Göttingen: Vandenhoeck \& Ruprecht.

Huibers, M. J. H., Lorenzo-Luaces, L., Cuijpers, P., \& Kazantzis, N. (2021). On the road to personalized psychotherapy: a research agenda based on cognitive behavior therapy for depression. frontiers in psychiatry 11. https://www. frontiersin.org/article/10.3389/fpsyt.2020.607508.Zugegriffen: 19. Juni 2021.

Jonas, B. (2020). Wirkfaktoren internetbasierter Beratungsinterventionen. Dissertation. Hildesheim: Fachbereich 1 Erziehungs- und Sozialwissenschaften der Universität.

Justen-Horsten, A., Paschen, H., (2016). Online-Interventionen in Therapie und Beratung. Ein Praxisleitfaden. Weinheim: Beltz.

Klasen, M., Knaevelsrud, C., \& Böttche, M. (2013). Die therapeutische Beziehung in internetbasierten Therapieverfahren: Ein Überblick. Der Nervenarzt, 84(7), 823-831. https://doi.org/10.1007/s00115-012-3659-6.

Knaevelsrud, C., Wagner, B., \& Böttche, M. (2016). OnlineTherapie und -Beratung. Ein Praxisleitfaden zur onlinebasierten Behandlung psychischer Störungen. Göttingen: Hogrefe.
Knatz, B. (2013). Handbuch Internetseelsorge. Grundlagen, Formen, Praxis. Gütersloh: Gütersloher Verlagshaus.

Knatz, B., \& Dodier, B. (2003). Hilfe aus dem Netz. Theorie und Praxis der Beratung per E-Mail. Stuttgart: Klett-Cotta.

Knatz, B., Dodier, B. (2021). Mailen, chatten, zoomen: Digitale Beratungsformen in der Praxis. Stuttgart: Klett-Cotta

Krieger, A., Winter, H., Müller, U.A., Ochs, M. \& Broicher, W. (Hrsg). (2015). Geht die Psychotherapie ins Netz? Möglichkeiten und Probleme von Therapie und Beratung im Internet. Gießen: Psychosozial-Verlag

Kühne, S., \& Hintenberger, G. (Hrsg.). (2009). Handbuch Online-Beratung. Göttingen:Vandenhoeck \& Ruprecht.

Kühne, S., \& Hintenberger, G. (2020). Onlineberatung und -therapie in Zeiten der Krise. Ein Überblick. e-beratungsjournal.net. Fachzeitschrift für Onlineberatung und computervermittelte Kommunikation 16(1), 33-45. https:// www.e-beratungsjournal.net/wp-content/uploads/ 2020/03/kuehne_hintenberger.pdf. Zugegriffen: 19. Juni 2021.

Maercker, A., Hecker, T., \& Heim, E. (2015). Personalisierte Internet-Psychotherapie-Angebote für die posttraumatische Belastungsstörung. Der Nervenarzt, 86(11), 1333-1342.

Meinlschmidt, G., \&Tegethoff, M. (2017). Psychotherapie:Quo vadis? Fortschritte der Neurologie. Psychiatrie, 85(08), 479-494.

Pennebaker, J.W. (1997). Writing about emotional experiences as a therapeutic process. Psychological Science, 8(3), 162-166. https://doi.org/10.1111/j.1467-9280.1997. tb00403.x.

Pfammatter, M., Junghan, U. M., \& Tschacher, W. (2012). Allgemeine Wirkfaktoren der Psychotherapie: Konzepte, Widersprüche und eine Synthese. Psychotherapie, 17(1), $17-31$.

Ploil, E. O. (2009). Psychosoziale Onlineberatung. München: Ernst Reinhardt Verlag

Probst, G. H., Berger, T., \& Flückiger, C. (2019). Die Allianz als Prädiktor für den Therapieerfolg internetbasierter Interventionen bei psychischen Störungen: Eine korrelative Metaanalyse. Verhaltenstherapie, 29, 182-195. https:// doi.org/10.1159/000501565.

Reindl, R. (2018). Zum Stand der Onlineberatung in Zeiten der Digitalisierung. e-beratungsjournal.net. Fachzeitschrift für Onlineberatung und computervermittelte Коттиnikation 14(1), 16-26. https://www.e-beratungsjournal. net/wp-content/uploads/2018/03/reindl.pdf. Zugegriffen: 19. Juni 2021.

Rubel, J., \& Lutz, W. (2016). „Personalisierung statt Verfahrensinflation" - Oder: Warum die Psychotherapieforschung in Deutschland ausgebaut werden sollte/könnte/müsste? Teil 2. Psychotherapie, Psychosomatik, Medizinische Psychologie, 66(12), 463-464.

Sack, M. (2019). Individualisierte Psychotherapie. Ein methodenübergreifendes Behandlungskonzept. Stuttgart:Schattauer.

Schultze,N. G. (2007).Erfolgsfaktoren des virtuellenSettingsin derpsychologischenInternet-Beratung.e-beratungsjournal.net - Fachzeitschrift für Online-Beratung und computervermittelte Kommunikation 4(1), 1-8. https:/ / www. e-beratungsjournal.net/ausgabe_0107/schultze.pdf.Zugegriffen: 19. Juni 2021.

Sieper, J. (2010). „Transversale Integration“: ein Kernkonzept der Integrativen Therapie - Einladung zu ko-respondierendem Diskurs. https://www.fpi-publikation.de/ download/10716/.Zugegriffen: 19. Juni2021.

Smoktunowicz, E., Barak, A., Andersson, G., Banos, R.M., Berger, T., Botella, C., Dear, B. F., Donker, T., Ebert, D. D., 


\section{übersichtsarbeit}

Hadjistavropoulos, H., Hodgins, D.C., Kaldo, V., Mohr, D. C., Nordgreen, T., Powers, M.P., Riper, H., Ritterband, L. M., Rozental, A., Schueller, S. M., Titov, N., Weise, C., \& Carlbring, P. (2020). Consensus statement on the problem of terminology in psychological interventions using the internet or digital components. Internet Interventions. https://doi.org/10.1016/j.invent.2020.100331.

Suler, J. (2004). The online disinhibition effect. CyberPsychology and Behavior, 7(3), 321-326. https://doi.org/10.1089/ 1094931041291295.

Wagner, B., \&Lange, A. (2008). InternetbasiertePsychotherapie „Interapy“. In S. Bauer \& H. Kordy (Hrsg.), E-MentalHealth (S. 105-120). Berlin: Springer.

Wagner, B., Horn, A. B., \& Maercker, A. (2014). Internet-based versus face-to-face cognitive-behavioral intervention for depression:Arandomized controllednon-inferiority trial. Journal of Affective Disorders, 152-154, 113-121. https:// doi.org/10.1016/j.jad.2013.06.032.
Wenzel, J. (2013). Wandel der Beratung durch Neue Medien. Göttingen:V\&Runipress

Wenzel, J. (2015). Mythos Unmittelbarkeit im Face-to-FaceKontakt - Weiterentwicklung von Beratung und Therapie durch gezielte methodische Nutzung der Medien e-beratungsjournal.net. Fachzeitschrift für Onlineberatung und computervermittelte Kommunikation 11(1), 36-54. https://www.e-beratungsjournal.net/ausgabe_ 0115/wenzel.pdf.Zugegriffen: 19. Juni2021.

Zwerenz, R., Becker, J., Johansson, R., Frederick, R., Andersson, G., \& Beutel, M. (2017). Transdiagnostic, psychodynamic web-based self-help intervention following inpatient psychotherapy: results of a feasibility study and randomized controlled trial. JMIR Ment Health. https://doi.org/ $10.2196 /$ mental.7889.

Hinweis des Verlags Der Verlag bleibt in Hinblick auf geografische Zuordnungen und Gebietsbezeichnungen in veröffentlichten Karten und Institutsadressen neutral. 